\title{
Xiphinema simile (Nematoda: Longidoridae) in the Czech Republic and a note on other Xiphinema species
}

\author{
S. KUMARI \\ Research Institute of Crop Production, Division of Plant Medicine, Drnovská 507, Ruzyně, 16106 Prague 6, Czech \\ Republic, E-mail: kumari@vurv.cz
}

\begin{abstract}
Summary
A nematode survey was carried out in South Moravia and Bohemia (Czech Republic) to assess the occurrence of $X i$ phinema in the rhizosphere of fruit orchards. Sixty six orchards in South Moravia and seven in Bohemia were studied during the years 2003 and 2004. Four Xiphinema species ( $X$. diversicaudatum, $X$. pachtaicum, $X$. simile and $X$. vuittenezi) were recorded. $X$. simile constitutes a first record for the nematodes fauna of the Czech Republic.
\end{abstract}

Key words: Xiphinema diversicaudatum; Xiphinema pachtaicum; Xiphinema simile; Xiphinema vuittenezi; PCR; Czech Republic

\section{Introduction}

Reports on the occurrence of Xiphinema in the territory of the Czech Republic date back to 1970s and late 1980s (Mali \& Vanek, 1972; Erbenová, 1973, 1975; Pelikán, 1989). After no attention was paid to nematodes of the genus Xiphinema and it was until recently (Kumari, 2004; Kumari et al., 2005) that problems of Xiphinema were put in the foreground again.

$X$. simile is a localised species in Europe (Lamberti et al., 2000) and it was reported from Bulgaria (Lamberti, et al., 1983; Peneva \& Choleva, 1992), Yugoslovakia (Barsi, 1994) and Slovakia (Lišková \& Brown, 1996). Outside Europe the species was recorded from Kenya (Coomans \& Heyns, 1997). The present study includes further record of $X$. simile in Europe and first record for the Czech fauna.

Several species of the genus Xiphinema include plant-parasitic nematodes and are natural vectors of economically important nepoviruses that cause substantial damage to a wide range of crops (Taylor \& Brown, 1997). Thus, accurate identification of those species that have the ability to transmit virus is essential. Problem associated with identification of Xiphinema on the bases of morphological and morphometrical characters that many characters overlap. Moreover, substantial morphological and morphometrical variability is apparent between the various species of Xiphinema. Recent studies have been demonstrated a great potential for molecular studies in Xiphinema species identification (Wang et al., 2003; Hübschen et al., 2004; Oliveira et al., 2005).

In this study, objectives were to:

(1) survey Xiphinema species occurring in the different orchards of South Moravia and Bohemia,

(2) identify different species of Xiphinema by morphological and morphometrical characters,

(3) conduct PCR-based methodology to verify the identification of $X$. diversicaudatum and $X$. vuittenezi.

\section{Material and Methods}

Morphological and morphometrical study

Nematode survey was carried out in the different regions of South Moravia and Bohemia, Czech Republic. Soil samples were taken at a depth of $0-90 \mathrm{~cm}$. Nematodes were recovered from the soil by sieving and decanting technique, heat killed, fixed in TAF, processed in slow glycerin process and mounted in anhydrous glycerin on slides. Photomicrographs were recorded with a digital camera linked to a computer and measurements were made with the aid of imaging software (Olympus DP-soft).

\section{Molecular study}

Total genomic DNA was extracted according to Hübschen et al. (2004) and DNA template was amplified by PCR using established primers by Wang et al. (2003). PCR reaction and gel electrophoresis condition were same as described by Kumari et al. (2005).

\section{Results}

Xiphinema simile Lamberti, Choleva et Agostinelli, 1983

Fig.1; Table 1, 2

$X$. simile was recorded at four (Čejkovice - under the rhi- 


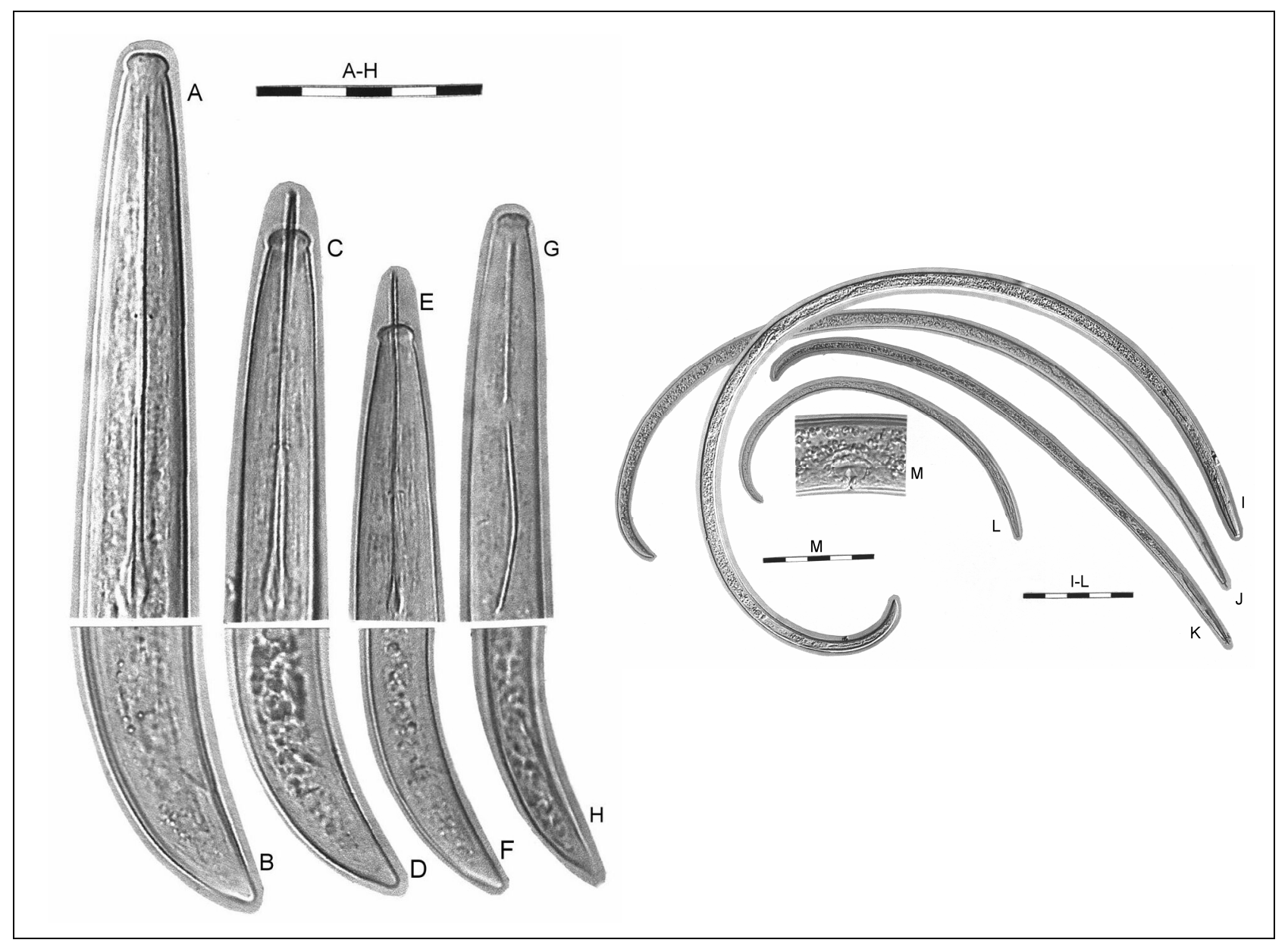

Fig. 1. Xiphinema simile Lamberti, Choleva et Agostinelli, 1983

A - H. Anterior and posterior regions. A, B: Female; C, D: JIII; E, F: JII; G, H: JI. I - L Entire nematodes. I: Female; J: JIII; K: JII; L: JI. M: Vulva. Scale bars: A - H, M, $50 \mu \mathrm{m} ; \mathrm{I}-\mathrm{L}, 200 \mu \mathrm{m}$

zosphere of sour cherry; Dětkovice - under the rhizosphere of sweet cherry; Prušánky - under the rhizosphere of apple; Velké Pavlovice - under the rhizosphere of apricot) of the 73 sampling orchards and occurred with population density of 4 to 25 adult females and 2 to 18 juveniles per $500 \mathrm{~g}$ soil. Adult females and juveniles were present at all four orchards, but not a single male was found. When adult females compared with Bulgarian (Peneva \& Choleva, 1992), Slovakian (Lišková \& Brown, 1996) and Kenyan (Coomans \& Heyns, 1997) populations, all morphometrics were closely agree except body length and pharyngeal bulb length. Mean body length of Czech population is similar to Slovakian populations but longer than Bulgarian and as well as Kenyan populations. Pharyngeal bulb length is longer than Kenyan population. Morphometrics of adult females also agree with the paratypes (Lamberti et al., 1983) except longer length of oral aperture to guide ring $(51 \mu \mathrm{m}$ vs. $60 \mu \mathrm{m} ; 58 \mu \mathrm{m} ; 60 \mu \mathrm{m} ; 59 \mu \mathrm{m})$. Beside some morphometrical variability, specimens from four populations appear to be morphologically similar.

According to Coomans and Heyns (1997) and Lamberti et al. (2000) the species has only three juvenile development stages. After comparing the body length, functional and replacement odontostyle length, distance of guiding ring from oral aperture and tail shape our data also confirmed the presence of three juvenile stages, with good agreement between the replacement and functional odontostyle length of successive stages. Juveniles were similar to females except for length and the more slender tail which gradually becomes thicker after each moult. JIII have a short conoid tail as in the female, whereas the two other juvenile stages have a longer slender tail. Juveniles of Czech population from Dětkovice and Velké Pavlovice correspond well with juvenile stages described from Kenya (Coomans \& Heyns, 1997).

26 orchards and vineyards (Kumari, 2004), 29 vineyards (Kumari et al., 2005) and 73 orchards (present study) have been surveyed during the years 2002 to 2004. A total of 128 sites have been surveyed and $X$. simile was recorded only from four sites.

Xiphinema diversicaudatum (Micoletzky, 1927) Thorne, 1939

Xiphinema pachtaicum (Tulaganov, 1938) Kirjanova, 1951 
Table 1. Morphometrics of three juvenile stages of Xiphinema simile Lamberti, Choleva et Agostinelli, 1983. Measurements in $\mu$ m (in form): mean \pm standard deviation (range)

\begin{tabular}{|c|c|c|c|c|c|}
\hline $\begin{array}{l}\text { Locality } \\
\text { Host }\end{array}$ & $\begin{array}{l}\text { Dětkovice } \\
\text { sweet cherry }\end{array}$ & $\begin{array}{c}\text { Dětkovice } \\
\text { sweet cherry }\end{array}$ & $\begin{array}{l}\text { Velké Pavlovice } \\
\text { apricot }\end{array}$ & $\begin{array}{c}\text { Dětkovice } \\
\text { sweet cherry }\end{array}$ & $\begin{array}{c}\text { Velké Pavlovice } \\
\text { apricot }\end{array}$ \\
\hline Specimens & JI & JII & JII & JIII & JIII \\
\hline $\mathrm{n}$ & 3 & 5 & 3 & 7 & 16 \\
\hline $\mathrm{L}$ & $\begin{array}{c}864 \pm 96 \\
(797-974)\end{array}$ & $\begin{array}{c}1267 \pm 100 \\
(1143-1420)\end{array}$ & $\begin{array}{c}1145 \pm 48 \\
(1105-1199)\end{array}$ & $\begin{array}{c}1803 \pm 174 \\
(1435-1975)\end{array}$ & $\begin{array}{l}1604 \pm 84 \\
(1386-1711)\end{array}$ \\
\hline $\mathrm{a}$ & $\begin{array}{c}54.8 \pm 5.35 \\
(48.7-58.7)\end{array}$ & $\begin{array}{c}56.1 \pm 4.0 \\
(52.4-61.7)\end{array}$ & $\begin{array}{c}53 \pm 9.28 \\
(42.5-60.0)\end{array}$ & $\begin{array}{c}66.1 \pm 5.5 \\
(58.4-74.9)\end{array}$ & $\begin{array}{c}62.6 \pm 4.54 \\
(53.9-70.3)\end{array}$ \\
\hline b & - & $\begin{array}{c}6.0 \pm 0.5 \\
(5.3-6.7)\end{array}$ & $\begin{array}{c}5.2 \pm 0.35 \\
(4.8-5.4)\end{array}$ & $\begin{array}{c}6.9 \pm 1.0 \\
(5.5-8.1)\end{array}$ & $\begin{array}{r}6.2 \pm 0.41 \\
(5.4-6.8)\end{array}$ \\
\hline $\mathrm{c}$ & - & $\begin{array}{c}41.5 \pm 4.5 \\
(36.4-48.4)\end{array}$ & $\begin{array}{c}41.4 \pm 2.11 \\
(39.0-42.8)\end{array}$ & $\begin{array}{c}54.3 \pm 6.4 \\
(49.3-68.1)\end{array}$ & $\begin{array}{c}52.6 \pm 5.37 \\
(43.2-61.3)\end{array}$ \\
\hline$c^{\prime}$ & - & $\begin{array}{c}2.24 \pm 0.23 \\
(2.00-2.61)\end{array}$ & $\begin{array}{c}2.04 \pm 0.30 \\
(1.70-2.30)\end{array}$ & $\begin{array}{c}1.93 \pm 0.16 \\
(1.70-2.23)\end{array}$ & $\begin{array}{c}1.80 \pm 0.15 \\
(1.62-2.07)\end{array}$ \\
\hline Replacement odontostyle & $\begin{array}{l}44 \pm 1.15 \\
(43-45)\end{array}$ & $\begin{array}{c}54 \pm 2.61 \\
(50-57)\end{array}$ & $\begin{array}{c}56 \pm 2.08 \\
(54-58)\end{array}$ & $\begin{array}{c}67 \pm 2.36 \\
(64-71)\end{array}$ & $\begin{array}{c}69 \pm 1.78 \\
(65-72)\end{array}$ \\
\hline Odontostyle & $\begin{array}{l}35 \pm 2.08 \\
(33-37)\end{array}$ & $\begin{array}{r}44 \pm 1.3 \\
(43-46)\end{array}$ & $\begin{array}{l}45 \pm 0.58 \\
(44-45)\end{array}$ & $\begin{array}{l}54 \pm 1.07 \\
(52-55)\end{array}$ & $\begin{array}{c}57 \pm 1.82 \\
(54-60)\end{array}$ \\
\hline Odontophore & $\begin{array}{l}29 \pm 1.52 \\
(28-31)\end{array}$ & $\begin{array}{l}34 \pm 2.86 \\
(30-38)\end{array}$ & $\begin{array}{l}31 \pm 3.46 \\
(27-33)\end{array}$ & $\begin{array}{c}36 \pm 1.6 \\
(33-38)\end{array}$ & $\begin{array}{l}36 \pm 1.61 \\
(33-39)\end{array}$ \\
\hline Total stylet length & $\begin{array}{l}65 \pm 0.58 \\
(64-65)\end{array}$ & $\begin{array}{l}78 \pm 3.53 \\
(75-84)\end{array}$ & $\begin{array}{l}76 \pm 3.21 \\
(72-78)\end{array}$ & $\begin{array}{l}90 \pm 1.95 \\
(86-92)\end{array}$ & $\begin{array}{l}93 \pm 2.25 \\
(90-98)\end{array}$ \\
\hline Greatest flange width & - & $\begin{array}{l}4 \pm 0.45 \\
(4-5)\end{array}$ & $\begin{array}{l}4 \pm 0.58 \\
(4-5)\end{array}$ & $\begin{array}{l}4 \pm 0.97 \\
(3-6)\end{array}$ & $\begin{array}{l}5 \pm 0.72 \\
(4-7)\end{array}$ \\
\hline Oral aperture to guide ring & $\begin{array}{l}30 \pm 1.73 \\
(28-31)\end{array}$ & $\begin{array}{c}38 \pm 1.3 \\
(37-40)\end{array}$ & $\begin{array}{l}36 \pm 1.15 \\
(35-37)\end{array}$ & $\begin{array}{l}47 \pm 2.88 \\
(43-50)\end{array}$ & $\begin{array}{l}46 \pm 1.76 \\
(44-50)\end{array}$ \\
\hline Pharyngeal bulb length & - & $\begin{array}{l}60 \pm 1.52 \\
(58-62)\end{array}$ & $\begin{array}{l}54 \pm 2.00 \\
(52-56)\end{array}$ & $\begin{array}{l}72 \pm 6.57 \\
(61-83)\end{array}$ & $\begin{array}{l}65 \pm 5.94 \\
(54-74)\end{array}$ \\
\hline Pharyngeal bulb diam. & - & $\begin{array}{c}11 \pm 1.14 \\
(9-12)\end{array}$ & $\begin{array}{l}12 \pm 1.00 \\
(11-13)\end{array}$ & $\begin{array}{c}13 \pm 1.73 \\
(11-16)\end{array}$ & $\begin{array}{l}13 \pm 1.41 \\
(11-16)\end{array}$ \\
\hline Tail length & - & $\begin{array}{l}31 \pm 3.96 \\
(26-34)\end{array}$ & $\begin{array}{l}28 \pm 1.53 \\
(26-29)\end{array}$ & $\begin{array}{l}33 \pm 3.91 \\
(28-38)\end{array}$ & $\begin{array}{l}31 \pm 3.34 \\
(26-39)\end{array}$ \\
\hline Length of hyaline tip & $\begin{array}{l}5 \pm 1.53 \\
(4-7)\end{array}$ & $\begin{array}{l}5 \pm 1.09 \\
(4-6)\end{array}$ & $\begin{array}{l}5 \pm 0.58 \\
(5-6)\end{array}$ & $\begin{array}{l}6 \pm 1.89 \\
(4-8)\end{array}$ & $\begin{array}{l}6 \pm 1.02 \\
(3-7)\end{array}$ \\
\hline Body diameter at lip region & $\begin{array}{l}7 \pm 0.58 \\
(7-8)\end{array}$ & $\begin{array}{l}8 \pm 0.56 \\
(7-8)\end{array}$ & $\begin{array}{l}8 \pm 0.58 \\
(7-8)\end{array}$ & $\begin{array}{l}8 \pm 0.58 \\
(7-9)\end{array}$ & $\begin{array}{l}9 \pm 0.63 \\
(8-10)\end{array}$ \\
\hline at guiding ring & $\begin{array}{l}13 \pm 0.58 \\
(13-14)\end{array}$ & $\begin{array}{l}15 \pm 0.84 \\
(14-16)\end{array}$ & $\begin{array}{l}16 \pm 2.64 \\
(14-19)\end{array}$ & $\begin{array}{l}17 \pm 0.53 \\
(17-18)\end{array}$ & $\begin{array}{l}17 \pm 0.82 \\
(16-18)\end{array}$ \\
\hline at base of pharynx & - & $\begin{array}{c}20 \pm 1.09 \\
(19-21)\end{array}$ & $\begin{array}{l}21 \pm 3.21 \\
(19-25)\end{array}$ & $\begin{array}{l}25 \pm 2.67 \\
(20-28)\end{array}$ & $\begin{array}{l}23 \pm 1.71 \\
(20-27)\end{array}$ \\
\hline at mid body & $\begin{array}{l}16 \pm 3.46 \\
(14-20)\end{array}$ & $\begin{array}{l}23 \pm 1.52 \\
(21-24)\end{array}$ & $\begin{array}{l}22 \pm 3.46 \\
(20-26)\end{array}$ & $\begin{array}{l}27 \pm 3.41 \\
(21-31)\end{array}$ & $\begin{array}{l}26 \pm 2.46 \\
(21-29)\end{array}$ \\
\hline at anus & - & $\begin{array}{l}14 \pm 1.64 \\
(12-16)\end{array}$ & $\begin{array}{l}14 \pm 1.53 \\
(12-15)\end{array}$ & $\begin{array}{l}17 \pm 1.25 \\
(15-19)\end{array}$ & $\begin{array}{l}17 \pm 1.41 \\
(14-19)\end{array}$ \\
\hline at beginning of hyaline tip & $\begin{array}{l}4 \pm 1.15 \\
(3-5)\end{array}$ & $\begin{array}{l}6 \pm 0.45 \\
(5-6)\end{array}$ & $\begin{array}{l}6 \pm 0.58 \\
(5-6)\end{array}$ & $\begin{array}{l}7 \pm 1.46 \\
(5-9)\end{array}$ & $\begin{array}{l}7 \pm 0.73 \\
(6-8)\end{array}$ \\
\hline
\end{tabular}

Xiphinema vuittenezi Luc, Lima, Weischer et Flegg, 1964 Figs. 2, 3; Tables 2, 3

Detail description of these three species from the Czech Republic was already given by Kumari (2004) and Kumari et al. (2005). Here only additional data is presented.

$X$. diversicaudatum was recorded in three orchards (peach, plum and sour cherry) at Bílé podolí, Bohemia out of the
73 sampling orchards and occurred with population density of 4 to 34 adult females, 4 to 42 adult males and 8 to 200 juveniles per $500 \mathrm{~g}$ soil. Between adult females one female specimen has all morphometrics similar to fourth stage juvenile (Table. 3). X. diversicaudatum was not found in South Moravia under rhizosphere of any orchard, but it was recorded in viticulture from South Moravia (Kumari et 


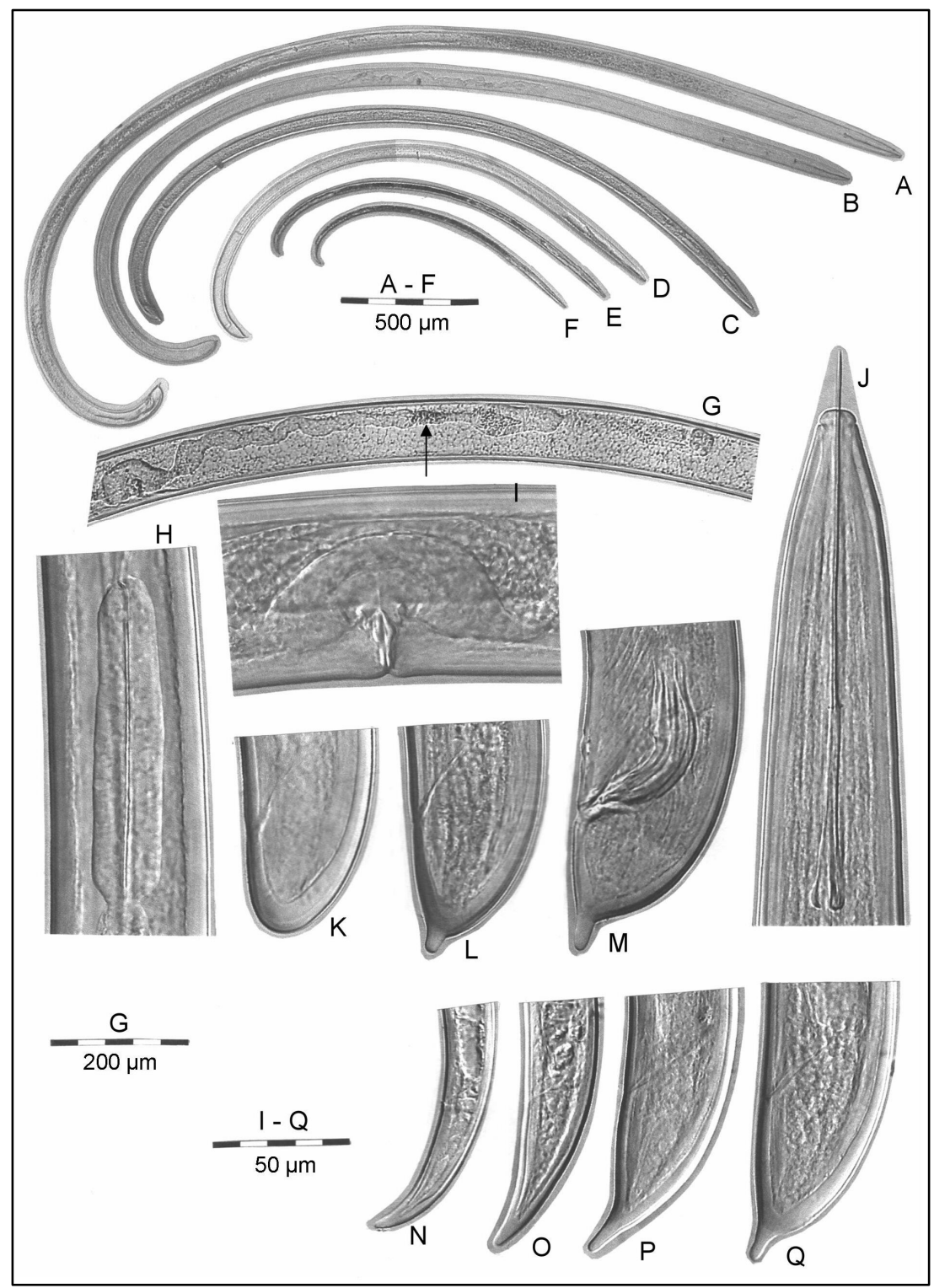

Fig. 2. Xiphinema diversicaudatum (Micoletzky, 1927) Thorne, 1939

A - F Entire nematodes. A: Male; B: Female; C: J4; D: J3; E: J2; F: J1. G: Posterior genital branch (arrow indicating Z-organ); H: Female pharyngeal bulb; I: Vulva; J: Female anterior; K, L: Female tail; M: Male tail; N: J1 tail; O: J2 tail; P: J3 tail: Q: J4 tail

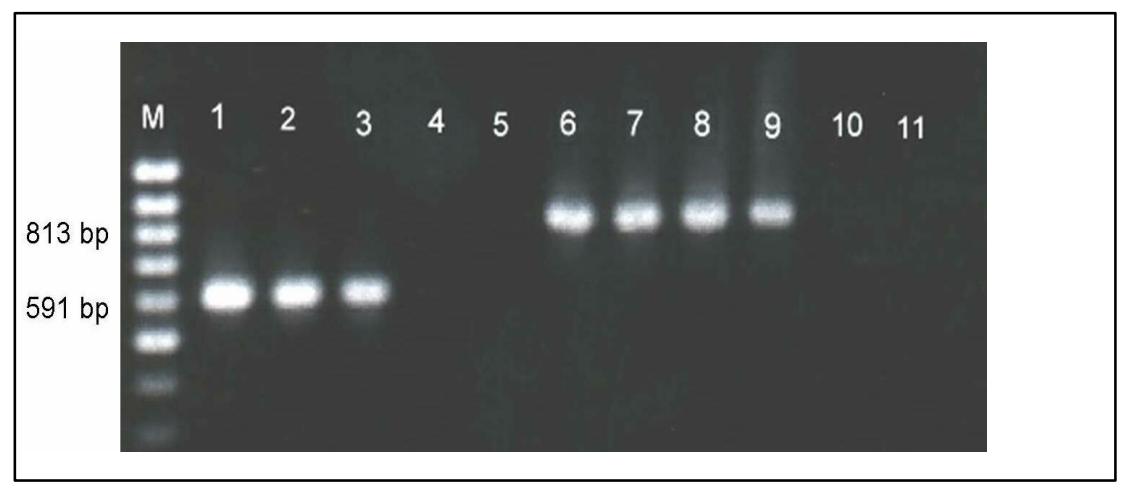

Fig. 3. Electrophoresis of the amplified products from single individual of Xiphinema vuittenezi and X. diversicaudatum. Lane M: 100 bp DNA ladder; lane 1 to $3=X$. vuittenezi (1: Čejkovice; 2: Hodonice; 3 : Kobylí); lane 4 negative control without DNA; lane 5: negative control with DNA (X. diversicaudastum); lane 6 to $9=X$. diversicaudatum (6: Bílé podolí (peach) for single female; 7: Bílé podolí (plum) for single female; 8 and 9 Bílé podolí (sour cherry) for single female and male; lane 10: negative control without DNA; lane 11: negative control with DNA (X. vuittenezi) 
Table 2. Morphometrics of adult females of Xiphinema simile Lamberti, Choleva et Agostinelli, 1983 and Xiphinema pachtaicum (Tulaganov, 1938) Kirjanova, 1951. Measurements in $\mu \mathrm{m}$ (in form): mean \pm standard deviation (range)

\begin{tabular}{|c|c|c|c|c|c|c|}
\hline \multirow{2}{*}{$\begin{array}{l}\text { Locality } \\
\text { Host } \\
\text { Species } \\
\end{array}$} & $\begin{array}{c}\text { Čejkovice } \\
\text { sour cherry }\end{array}$ & $\begin{array}{c}\text { Dětkovice } \\
\text { sweet cherry }\end{array}$ & \multicolumn{2}{|c|}{$\begin{array}{c}\text { Prušánky } \\
\text { apple }\end{array}$} & $\begin{array}{c}\text { Velké Pavlovice } \\
\text { apricot }\end{array}$ & \multirow{2}{*}{$\begin{array}{c}\begin{array}{c}\text { Mohyla míru } \\
\text { apple }\end{array} \\
\text { X. pachtaicum } \\
\end{array}$} \\
\hline & \multicolumn{5}{|c|}{$X$. simile } & \\
\hline $\mathrm{n}$ & 20 & 22 & & & 48 & 5 \\
\hline $\mathrm{L}$ & $\begin{array}{c}2175 \pm 158 \\
(1899-2513)\end{array}$ & $\begin{array}{c}2293 \pm 114 \\
(2035-2487)\end{array}$ & 2243 & 1974 & $\begin{array}{c}2210 \pm 155 \\
(1838-2613)\end{array}$ & $\begin{array}{l}2081 \pm 92 \\
(1988-2232)\end{array}$ \\
\hline $\mathrm{a}$ & $\begin{array}{c}63.0 \pm 7.56 \\
(54.0-80.5)\end{array}$ & $\begin{array}{c}72.2 \pm 8.16 \\
(59-85)\end{array}$ & 64.1 & 71.0 & $\begin{array}{c}72.8 \pm 5.51 \\
(62.8-82.3)\end{array}$ & $\begin{array}{c}57.7 \pm 5.03 \\
(52.3-63.6)\end{array}$ \\
\hline b & $\begin{array}{r}7.5 \pm 0.47 \\
(6.7-8.3)\end{array}$ & $\begin{array}{r}7.7 \pm 0.72 \\
(6.6-9.5)\end{array}$ & 7.8 & 7.1 & $\begin{array}{c}7.5 \pm 0.95 \\
(5.8-10.7)\end{array}$ & $\begin{array}{r}7.7 \pm 0.58 \\
(7.2-8.7)\end{array}$ \\
\hline c & $\begin{array}{c}77.0 \pm 6.80 \\
(65.5-95.0)\end{array}$ & $\begin{array}{c}79 \pm 9.43 \\
(62.3-99.8)\end{array}$ & 70.1 & 70.5 & $\begin{array}{c}73.7 \pm 5.69 \\
(60.8-88.9)\end{array}$ & $\begin{array}{c}72.7 \pm 8.73 \\
(63.8-86.8)\end{array}$ \\
\hline $\mathrm{c}^{\prime}$ & $\begin{array}{c}1.56 \pm 0.15 \\
(1.22-1.76)\end{array}$ & $\begin{array}{c}1.65 \pm 0.20 \\
(1.35-2.19)\end{array}$ & 1.68 & 1.65 & $\begin{array}{c}1.65 \pm 0.15 \\
(1.03-2.05)\end{array}$ & $\begin{array}{c}1.74 \pm 0.18 \\
(1.50-1.94)\end{array}$ \\
\hline V & $\begin{array}{c}54 \pm 0.91 \\
(52-56)\end{array}$ & $\begin{array}{c}54 \pm 1.30 \\
(51-57)\end{array}$ & 54 & 55 & $\begin{array}{c}55 \pm 1.36 \\
(50-58)\end{array}$ & $\begin{array}{c}54 \pm 1.3 \\
(53-56)\end{array}$ \\
\hline Odontostyle & $\begin{array}{l}67 \pm 1.87 \\
(61-69)\end{array}$ & $\begin{array}{l}67 \pm 1.91 \\
(62-70)\end{array}$ & 65 & 67 & $\begin{array}{l}68 \pm 2.33 \\
(62-73)\end{array}$ & $\begin{array}{l}79 \pm 2.07 \\
(77-82)\end{array}$ \\
\hline Odontophore & $\begin{array}{l}42 \pm 2.03 \\
(39-46)\end{array}$ & $\begin{array}{l}44 \pm 1.82 \\
(41-49)\end{array}$ & 41 & 40 & $\begin{array}{l}43 \pm 2.07 \\
(38-47)\end{array}$ & $\begin{array}{l}41 \pm 2.41 \\
(38-44)\end{array}$ \\
\hline Total stylet length & $\begin{array}{r}109 \pm 2.39 \\
(105-113)\end{array}$ & $\begin{array}{c}111 \pm 2.85 \\
(106-118)\end{array}$ & 106 & 107 & $\begin{array}{c}111 \pm 3.17 \\
(104-120)\end{array}$ & $\begin{array}{c}121 \pm 1.48 \\
(119-123)\end{array}$ \\
\hline Greatest flange width & $\begin{array}{l}5 \pm 0.64 \\
(4-7)\end{array}$ & $\begin{array}{l}6 \pm 0.74 \\
(4-7)\end{array}$ & 6 & 7 & $\begin{array}{l}6 \pm 0.71 \\
(4-7)\end{array}$ & $\begin{array}{c}6 \pm 0 \\
(6-6)\end{array}$ \\
\hline Oral aperture to guide ring & $\begin{array}{c}60 \pm 2.11 \\
(57-65)\end{array}$ & $\begin{array}{c}58 \pm 2.09 \\
(55-63)\end{array}$ & 60 & 60 & $\begin{array}{l}59 \pm 3.14 \\
(53-68)\end{array}$ & $\begin{array}{l}72 \pm 2.59 \\
(69-74)\end{array}$ \\
\hline Pharyngeal bulb length & $\begin{array}{l}82 \pm 3.44 \\
(77-90)\end{array}$ & $\begin{array}{l}86 \pm 5.28 \\
(72-90)\end{array}$ & 82 & 80 & $\begin{array}{l}80 \pm 4.97 \\
(67-90)\end{array}$ & $\begin{array}{l}60 \pm 2.88 \\
(56-63)\end{array}$ \\
\hline Pharyngeal bulb diam. & $\begin{array}{l}14 \pm 1.80 \\
(12-18)\end{array}$ & $\begin{array}{l}17 \pm 2.20 \\
(11-20)\end{array}$ & 13 & 13 & $\begin{array}{l}16 \pm 1.75 \\
(13-19)\end{array}$ & $\begin{array}{l}14 \pm 1.14 \\
(12-15)\end{array}$ \\
\hline Tail length & $\begin{array}{l}28 \pm 2.64 \\
(24-34)\end{array}$ & $\begin{array}{l}29 \pm 3.16 \\
(24-37)\end{array}$ & 32 & 28 & $\begin{array}{l}30 \pm 2.17 \\
(24-35)\end{array}$ & $\begin{array}{l}29 \pm 4.00 \\
(24-35)\end{array}$ \\
\hline Length of hyaline tip & $\begin{array}{l}4 \pm 0.98 \\
(2-6)\end{array}$ & $\begin{array}{l}7 \pm 1.27 \\
(4-9)\end{array}$ & 6 & 7 & $\begin{array}{l}7 \pm 1.27 \\
(5-10)\end{array}$ & $\begin{array}{c}8 \pm 2.07 \\
(6-11)\end{array}$ \\
\hline Body diameter at lip region & $\begin{array}{l}9 \pm 0.22 \\
(8-9)\end{array}$ & $\begin{array}{l}9 \pm 0.35 \\
(9-10)\end{array}$ & 10 & 9 & $\begin{array}{l}9 \pm 0.56 \\
(8-10)\end{array}$ & $\begin{array}{l}9 \pm 0.55 \\
(8-9)\end{array}$ \\
\hline at guiding ring & $\begin{array}{l}20 \pm 0.94 \\
(18-21)\end{array}$ & $\begin{array}{l}19 \pm 1.22 \\
(18-22)\end{array}$ & 19 & 19 & $\begin{array}{l}19 \pm 0.90 \\
(18-22)\end{array}$ & $\begin{array}{l}22 \pm 1.14 \\
(20-23)\end{array}$ \\
\hline at base of pharynx & $\begin{array}{l}27 \pm 2.70 \\
(23-31)\end{array}$ & $\begin{array}{l}28 \pm 3.08 \\
(23-34)\end{array}$ & 28 & 26 & $\begin{array}{l}26 \pm 1.43 \\
(23-29)\end{array}$ & $\begin{array}{l}29 \pm 1.92 \\
(26-31)\end{array}$ \\
\hline at vulva & $\begin{array}{l}35 \pm 4.88 \\
(25-42)\end{array}$ & $\begin{array}{l}32 \pm 4.54 \\
(25-39)\end{array}$ & 35 & 28 & $\begin{array}{l}31 \pm 2.88 \\
(26-39)\end{array}$ & $\begin{array}{l}36 \pm 2.68 \\
(32-39)\end{array}$ \\
\hline at anus & $\begin{array}{l}18 \pm 1.78 \\
(16-22)\end{array}$ & $\begin{array}{l}18 \pm 1.41 \\
(16-21)\end{array}$ & 19 & 17 & $\begin{array}{l}18 \pm 1.24 \\
(16-20)\end{array}$ & $\begin{array}{l}17 \pm 0.89 \\
(16-18)\end{array}$ \\
\hline at beginning of hyaline tip & $\begin{array}{l}6 \pm 0.60 \\
(5-7) \\
\end{array}$ & $\begin{array}{c}8 \pm 1.17 \\
(6-10) \\
\end{array}$ & 7 & 8 & $\begin{array}{c}9 \pm 1.17 \\
(4-11) \\
\end{array}$ & $\begin{array}{l}6 \pm 1.10 \\
(5-7) \\
\end{array}$ \\
\hline
\end{tabular}

al., 2005), where the species occurred with $X$. vuittenezi. The two species $X$. diversicaudatum and $X$. vuittenezi are so closely related that it was impossible to separate their juvenile stages with certainty. Therefore morphometrical data for juvenile stages was not studied previously from the Czech Republic. Here morphometrical data for all four juvenile stages has been presented from Bílé Podolí under the rhizosphere of sour cherry (Table 3 ). The four juvenile stages are recognised by the length of the body and of the functional and replacement odontostyle. The length of the replacement odontostyle of one stage corresponds to the length of the functional odontostyle of the next older stage. Only fourth stage juveniles have tails similar to adults, earlier stages have more tapering tails lacking a distinct peg (Fig.2). Morphometrics of juveniles of $X$. diversicaudatum were similar to juveniles from Novi Sad (Barsi \& 
Table 3. Morphometrics of Xiphinema diversicaudatum (Micoletzky, 1927) Thorne, 1939. Measurements in $\mu \mathrm{m}$ (in form): mean \pm standard deviation (range)

\begin{tabular}{|c|c|c|c|c|c|c|c|}
\hline \multirow{2}{*}{$\begin{array}{l}\text { Locality } \\
\text { Host } \\
\text { Specimens }\end{array}$} & \multicolumn{7}{|c|}{$\begin{array}{l}\text { Bílé Podolí } \\
\text { sour cherry }\end{array}$} \\
\hline & J1 & $\mathrm{J} 2$ & $\mathrm{~J} 3$ & $\mathrm{~J} 4$ & Females & $\begin{array}{r}\text { Female } \\
\text { (short) }\end{array}$ & Males \\
\hline $\mathrm{n}$ & 7 & 6 & 18 & 14 & 22 & 1 & 10 \\
\hline \multirow[t]{2}{*}{$\mathrm{L}$} & $1010 \pm 81$ & $1445 \pm 90$ & $2274 \pm 210$ & $3241 \pm 349$ & $4644 \pm 369$ & 3245 & $4591 \pm 307$ \\
\hline & $(873-1102)$ & $(1320-1550)$ & $(1817-2559)$ & $(2636-3859)$ & $(3974-5484)$ & & $(4154-5126)$ \\
\hline \multirow[t]{2}{*}{$\mathrm{a}$} & $42.6 \pm 2.80$ & $43.0 \pm 2.42$ & $51.9 \pm 4.15$ & $59.6 \pm 5.07$ & $64.5 \pm 3.25$ & 52.3 & $69.5 \pm 3.78$ \\
\hline & $(36.7-45.1)$ & $(39.6-45.6)$ & $(46.5-60.5)$ & $(52.0-71.2)$ & $(59.5-69.9)$ & & $(65.0-76.7)$ \\
\hline \multirow[t]{2}{*}{$\mathrm{b}$} & $4.1 \pm 0.40$ & $4.9 \pm 0.29$ & $5.9 \pm 0.59$ & $7.2 \pm 0.76$ & $9.5 \pm 0.93$ & 7.6 & $8.9 \pm 0.52$ \\
\hline & $(3.4-4.6)$ & $(4.6-5.3)$ & $(4.9-7.1)$ & $(6.1-8.3)$ & $(7.8-11.4)$ & & $(8.1-10.0)$ \\
\hline \multirow[t]{2}{*}{ c } & $18.2 \pm 1.14$ & $23.6 \pm 1.94$ & $40.0 \pm 5.91$ & $61.5 \pm 9.00$ & $102.6 \pm 8.95$ & 75.5 & $96.0 \pm 9.61$ \\
\hline & $(16.5-20.1)$ & $(20.6-26.3)$ & $(31.7-56.3)$ & $(48.0-78.8)$ & $(88.3-121.1)$ & & $(78.8-108.9)$ \\
\hline \multirow[t]{2}{*}{$c^{\prime}$} & $3.64 \pm 0.28$ & $2.71 \pm 0.32$ & $1.84 \pm 0.24$ & $1.31 \pm 0.13$ & $0.88 \pm 0.08$ & 0.91 & $0.99 \pm 0.06$ \\
\hline & $(3.36-4.07)$ & $(2.46-3.30)$ & $(1.40-2.21)$ & $(1.17-1.65)$ & $(0.73-1.02)$ & & $(0.92-1.09)$ \\
\hline Replacement & $67 \pm 2.27$ & $88 \pm 5.05$ & $111 \pm 5.32$ & $136 \pm 4.99$ & - & - & - \\
\hline odontostyle & $(62-69)$ & $(81-95)$ & $(97-118)$ & $(129-145)$ & & & \\
\hline \multirow[t]{2}{*}{ V/spicule } & - & - & - & - & $40.0 \pm 1.49$ & 42.5 & $74 \pm 5.09$ \\
\hline & & & & & $(37.7-43.2)$ & & $(67-85)$ \\
\hline \multirow[t]{2}{*}{ Odontostyle } & $51 \pm 1.38$ & $64 \pm 1.17$ & $87 \pm 3.12$ & $108 \pm 4.26$ & $132 \pm 4.69$ & 111 & $132 \pm 4.74$ \\
\hline & $(49-52)$ & $(63-66)$ & $(81-93)$ & $(99-113)$ & $(123-138)$ & & $(124-140)$ \\
\hline \multirow[t]{2}{*}{ Odontophore } & $37 \pm 1.90$ & $46 \pm 1.67$ & $57 \pm 2.06$ & $69 \pm 3.07$ & $81 \pm 3.11$ & 73 & $80 \pm 2.79$ \\
\hline & $(34-39)$ & $(44-49)$ & $(53-61)$ & $(63-73)$ & $(76-86)$ & & $(75-84)$ \\
\hline \multirow[t]{2}{*}{ Total stylet length } & $87 \pm 2.69$ & $110 \pm 2.32$ & $144 \pm 6.51$ & $178 \pm 5.87$ & $213 \pm 6.36$ & 184 & $212 \pm 5.87$ \\
\hline & $(83-90)$ & $(107-114)$ & $(125-154)$ & $(168-186)$ & $(200-224)$ & & $(202-220)$ \\
\hline \multirow[t]{2}{*}{ Greatest flange width } & $7 \pm 0.58$ & $9 \pm 0.55$ & $10 \pm 0.99$ & $11 \pm 0.73$ & $12 \pm 0.75$ & 13 & $12 \pm 1.37$ \\
\hline & $(6-8)$ & $(8-9)$ & $(8-11)$ & $(10-13)$ & $(11-14)$ & & $(9-14)$ \\
\hline \multirow{2}{*}{$\begin{array}{l}\text { Oral aperture to guide } \\
\text { ring }\end{array}$} & $41 \pm 1.83$ & $54 \pm 4.13$ & $79 \pm 5.45$ & $94 \pm 8.17$ & $120 \pm 9.31$ & 108 & $120 \pm 8.08$ \\
\hline & $(39-44)$ & $(50-62)$ & $(69-93)$ & $(78-107)$ & $(104-135)$ & & $(109-133)$ \\
\hline \multirow{2}{*}{$\begin{array}{l}\text { Pharyngeal bulb } \\
\text { length }\end{array}$} & $61 \pm 5.19$ & $72 \pm 4.68$ & $85 \pm 5.18$ & $96 \pm 6.59$ & $108 \pm 6.62$ & 102 & $109 \pm 5.59$ \\
\hline & $(51-65)$ & $(64-76)$ & $(69-91)$ & $(83-109)$ & $(94-118)$ & & $(102-119)$ \\
\hline \multirow[t]{2}{*}{ Pharyngeal bulb diam. } & $14 \pm 2.23$ & $20 \pm 1.83$ & $24 \pm 2.61$ & $27 \pm 2.83$ & $32 \pm 3.59$ & 34 & $31 \pm 2.84$ \\
\hline & $(12-18)$ & $(18-23)$ & $(18-28)$ & $(23-32)$ & $(26-40)$ & & $(25-35)$ \\
\hline \multirow[t]{2}{*}{ Tail length } & $56 \pm 4.47$ & $62 \pm 4.42$ & $57 \pm 5.07$ & $53 \pm 4.60$ & $45 \pm 4.19$ & 43 & $48 \pm 5.35$ \\
\hline & $(47-60)$ & $(55-66)$ & $(42-63)$ & $(48-61)$ & $(38-52)$ & & $(40-57)$ \\
\hline \multirow[t]{2}{*}{ Length of hyaline tip } & $9 \pm 1.11$ & $15 \pm 2.28$ & $19 \pm 2.32$ & $16 \pm 1.79$ & $16 \pm 2.68$ & 14 & $17 \pm 2.23$ \\
\hline & $(7-10)$ & $(11-18)$ & $(14-23)$ & $(14-19)$ & $(11-21)$ & & $(14-21)$ \\
\hline \multirow{4}{*}{$\begin{array}{l}\text { Body diam. at lip } \\
\text { region } \\
\text { at guiding ring }\end{array}$} & $7 \pm 0.53$ & $8 \pm 0.52$ & $10 \pm 0.84$ & $11 \pm 0.61$ & $13 \pm 0.75$ & 12 & $13 \pm 0.74$ \\
\hline & $(7-8)$ & $(8-9)$ & $(8-12)$ & $(10-12)$ & $(12-14)$ & & $(12-14)$ \\
\hline & $16 \pm 0.38$ & $23 \pm 1.94$ & $29 \pm 2.43$ & $35 \pm 3.20$ & $45 \pm 3.31$ & 40 & $42 \pm 4.01$ \\
\hline & $(16-17)$ & $(20-25)$ & $(26-35)$ & $(29-40)$ & $(40-50)$ & & $(36-47)$ \\
\hline \multirow[t]{2}{*}{ at base of pharynx } & $23 \pm 2.21$ & $32 \pm 3.01$ & $41 \pm 4.58$ & $51 \pm 6.96$ & $63 \pm 4.44$ & 56 & $59 \pm 5.38$ \\
\hline & $(20-27)$ & $(28-36)$ & $(29-48)$ & $(37-61)$ & $(56-70)$ & & $(50-66)$ \\
\hline \multirow[t]{2}{*}{ at mid body/at vulva } & $24 \pm 3.24$ & $34 \pm 2.66$ & $44 \pm 5.43$ & $55 \pm 7.27$ & $72 \pm 4.79$ & 62 & $66 \pm 5.07$ \\
\hline & $(20-30)$ & $(31-38)$ & $(30-54)$ & $(37-65)$ & $(64-83)$ & & $(58-73)$ \\
\hline \multirow[t]{2}{*}{ at anus } & $15 \pm 1.38$ & $23 \pm 1.94$ & $31 \pm 3.53$ & $41 \pm 3.46$ & $52 \pm 5.15$ & 47 & $48 \pm 3.62$ \\
\hline & $(13-17)$ & $(20-26)$ & $(25-39)$ & $(32-46)$ & $(42-63)$ & & $(42-53)$ \\
\hline at beginning of & $6 \pm 0.76$ & $8 \pm 0.82$ & $11 \pm 0.98$ & $17 \pm 1.45$ & $24 \pm 3.03$ & 22 & $24 \pm 4.11$ \\
\hline hyaline tip & $(6-8)$ & $(7-9)$ & $(9-13)$ & $(14-19)$ & $(19-28)$ & & $(18-32)$ \\
\hline
\end{tabular}

Lamberti, 2000), except greater body diameter and mean body length and replacement odontostyle length.

$X$. pachtaicum was recorded only at one orchard at Mohyla míru (South Moravia) associated with apple. Three samples were collected from the locality but only one sample was positive for $X$. pachtaicum with population density of 5 adult females and 11 juveniles. Male were not found.
$X$. vuitttenezi recorded at 60 different orchards out of 73 sampling orchards associated with apple, apricot, peach, plum, sour cherry and sweet cherry with population density of 2 to 124 adult females and 1 to 158 juveniles. Males were found at three orchards. At one of the localities (Lednice), within the population of $X$. vuittenezi one specimen was found with posterior vulva (Kumari, 2003). 
Molecular study of $X$. diversicaudatum and X. vuittenezi Using the established primers (Wang et al., 2003), classical identification of $X$. diversicaudatum and $X$. vuittenez $i$ was verified by PCR. All positive orchards for X. vuittenezi were verified by PCR but here data is shown only for three selected localities. PCR with species-specific primers yielded a single fragment of approximately 813 bp for $X$. diversicaudatum and 591 bp for $X$. vuittenezi (Fig.3). No PCR products were obtained in the negative control lacking DNA template and negative control containing DNA template of other Xiphinema species.

\section{Discussion}

In South Moravia and Bohemia 73 orchards were surveyed, other than previously surveyed orchards (Kumari, 2004). Four Xiphinema species $X$. diversicaudatum, $X$. pachtaicum, $X$. simile and $X$. vuittenezi were recorded. Three species of $X$. americanum-lineage (putative and established members) occurring in the Czech Republic are $X$. brevicollum, $X$. pachtaicum and $X$. simile. Two species $X$. simile and $X$. pachtaicum are morphologically similar. $X$. simile and $X$. pachtaicum can be distinguished from each other by their body and odontostyle length, distance of guiding ring from oral aperture and shape of vulva and tail. $X$. simile have a longer and slender body, shorter odontostyle and short distance of oral aperture to guiding ring. $X$. simile tail terminus is more rounded, where $X$. pachtaicum has more pointed tail. The shape of vulva is also different in both species.

As compare to other species of the genus Xiphinema $(X$. brevicollum, $X$. diversicaudatum, $X$. pachtaicum and $X$. vuittenezi) described from the Czech Republic, $X$. simile unequivocally has only three juvenile stages. $X$. vuittenezi was the most widespread species, which occurred in 60 out of 73 surveyed orchards.

It has been demonstrated that for routine identification of plant-parasitic nematodes, DNA based diagnostics are quicker than the traditional strategy using morphology and morphometrics (Wang et al., 2003; Hübschen et al., 2004; Oliveira et al., 2005), but morphological identification is still a prerequisite for DNA based diagnostics. Therefore, combination of molecular and morphological study can be the most reliable approach for the identification of Xiphinema species.

In the Czech Republic further comprehensive survey is necessary to study the occurrence and distribution of Xiphinema and it will be also important to study virus-vector relationship.

\section{Acknowledgements}

The author is thankful to R. Choutka, M. Chaloupková, M. Jokeš and F. Dvořák for assistance in collecting soil samples and L. Sýkorová for processing soil samples. The work was supported by the Ministry of Agriculture of the Czech Republic, Project number MZe - 0002700603.

\section{References}

BARSI, L. (1994): Species of the Xiphinema americanumgroup (Nematoda: Dorylaimida) on the territory of the former Yugoslavia. Nematol. Medit., 22: 25 - 34

BARSI, L., LAMBERTI, F. (2000): Morphometric variation and juvenile stages of Xiphinema diversicaudatum (Micoletzky, 1927) Thorne, 1939 and $X$. index Thorne et Allen, 1950 (Nematoda: Dorylaimida) from the former territory of Yugoslavia. Nematol. Medit., 28: 171 - 187

Coomans, A., Heyns, J. (1997): Three species of the $X i$ phinema americanum-group (Nematoda: Longidoridae) from Kenya. Nematologica, 43: 259 - 274

ERBENOVÁ, M. (1973): [Partial final report - Survey of the nematodes fauna in fruit orchards]. Research Institute of Pomology, Holovousy, former Czechoslovakia

ERBENOVÁ, M. (1975): [Ectoparasitic nematodes of the genus Xiphinema Cobb 1913 in orchards in Czechoslovakia]. Sb. ÚVTI - Zahradnictví, 2: $79-86$

HÜBSCHEN, J., Kling, L., IPACH, U., ZINKERNAGEL, V., Bosselut, N., Esmenjaud, D., Brown, D. J. F., NeILson, R. (2004): Validation of the specificity and sensitivity of species-specific primers that provide a reliable molecular diagnostic for Xiphinema diversicaudatum, $X$. index and $X$. vuittenezi. Eur. J. Pl. Path., 110: 779 - 788

KUMARI, S. (2003): A single specimen of Xiphinema vuittenezi with posterior vulva from the Czech Republic. Int. J. Nematol., 13: 233 - 235

KUMARI, S. (2004): The occurrence of Xiphinema vuittenezi, X. pachtaicum and Longidorus leptocephalus (Nematoda: Dorylaimida) in the Central Czech Republic. Helminthologia, 41: $103-108$

Kumari, S., PolÁK, J., ChoutKA, R. (2005): Plant-parasitic nematodes of the genus Xiphinema (Nematoda: Longidoridae) in the vineyards of the Czech Republic. Nematology, 7: $81-93$

Lamberti, F., Choleva, B., Agostinelli, A. (1983): Longidoridae from Bulgaria (Nematoda, Dorylaimida) with description of three new species of Longidorus and two new species of Xiphinema. Nematol. Medit., 11: $49-$ 72

LAmberti, F., Molinari, S., Brown, D. J. F. (2000): The Xiphinema americanum group. I. Putative species, their geographical occurrence and distribution, and regional polytomous identification keys for the group. Russ. J. Nematol., 8: $65-84$

LiŠKOVÁ, M., BROWN, D. J. F. (1996): Taxonomic validity and ecological relations of Xiphinema pachtaicum and $X$. simile (Nematoda: Dorylaimida), two members of the $X$. americanum group occurring in Slovakia. Helminthologia, 33: $137-142$

MaLI, V. R., VANEK, G. (1972): Nematode of the family Longidoridae (Thorne) Meyl and their role in Czechoslovakian vineyards. Biológia, 27, 11: $841-852$

Oliveira, C. M. G., Fenton, B., Malloch, G., Brown, D. J. F., NeILSON, R. (2005): Development of species-specific primers for the ectoparasitic nematode species Xiphinema brevicolle, $X$. diffusum, $X$. elongatum, $X$. ifacolum 
and $X$. longicaudatum (Nematoda: Longidoridae) based on ribosomal DNA sequences. Ann. App. Biol., 146: 281 288

PELIKÁN, E. (1989): Plant viruses and nematodes as virus vectors in the ecosystems of South Bohemia. Proceedings of the $10^{\text {th }}$ conference of the Czechoslovak plant virologists (Abst.): 148

Peneva, V., Choleva, B. (1992): Nematodes of the family Longidoridae from forest nurseries in Bulgaria. II. The

RECEIVED JUNE 30, 2005 genus Xiphinema Cobb, 1913. Helminthology, 32: 46 - 58 TAYlor, C. E., Brown, D. J. F. (1997): Nematode vectors of plant viruses. CABI, UK

Wang, X., Bosselut, N., Castagnone, C., Voisin, R., ABAD, P., Esmenjaud, D. (2003): Multiplex polymerase chain reaction identification of single individuals of the longidorid nematodes Xiphinema index, $X$. diversicaudatum, $X$. vuittenezi, and $X$. italiae using specific primers from ribosomal genes. Phytopathology, 93: 160 - 166 